\title{
Correction to: Racial/Ethnic Disparities in Opioid-Related Mortality in the USA, 1999-2019: the Extreme Case of Washington DC
}

\author{
Mathew V. Kiang • Alexander C. Tsai • \\ Monica J. Alexander · David H. Rehkopf • \\ Sanjay Basu
}

Published online: 19 November 2021

(C) The New York Academy of Medicine 2021

\section{Correction to: J Urban Health (2021) 98:589-595 https://doi.org/10.1007/s11524-021-00573-8}

Due to an error during the production process, the abstract and key words were missing from this article as originally published.

The original article has been corrected.

Publisher's Note Springer Nature remains neutral with regard to jurisdictional claims in published maps and institutional affiliations.

The original article can be found online at https://doi.org/ 10.1007/s11524-021-00573-8

M. V. Kiang $(\bowtie) \cdot$ D. H. Rehkopf

Department of Epidemiology and Population Health, Stanford University School of Medicine, Stanford, CA, USA e-mail: mkiang@stanford.edu

\section{V. Kiang}

Harvard FXB Center for Health and Human Rights,

Boston, MA, USA

\section{V. Kiang}

Center for Population Health Sciences, Stanford University,

1701 Page Mill Road, Palo Alto, CA 94304, USA

\section{A. C. Tsai}

Center for Global Health and Mongan Institute,

Massachusetts General Hospital, Boston, MA, USA

\section{A. C. Tsai}

Harvard Medical School, Boston, MA, USA
M. J. Alexander

Department of Statistical Sciences, University of Toronto, Toronto, ON, Canada

M. J. Alexander

Department of Sociology, University of Toronto, Toronto, ON, Canada

D. H. Rehkopf

Division of Primary Care and Population Health, Stanford University School of Medicine, Stanford, CA, USA

S. Basu

Institute of Health Policy, Management, and Evaluation, University of Toronto, Toronto, ON, Canada

S. Basu

School of Public Health, Imperial College, London, UK

S. Basu

Center for Primary Care, Harvard Medical School, Boston, MA, USA 